\title{
Collateral circulation formation determines the characteristic profiles of contrast-enhanced MRI in the infarcted myocardium of pigs
}

\author{
Jian WANG ${ }^{1,2,3, *}$, Bo XIANG ${ }^{2,3}$, Hung-yu LIN², Hong-yu LIU4 ${ }^{4}$, Darren FREED ${ }^{2,3,5}$, Rakesh C ARORA ${ }^{2,3,5}$, Gang-hong TIAN TI $^{2,3}$ \\ ${ }^{1}$ Department of Vascular Surgery, Union Hospital, Tongji Medical College, Huazhong University of Science and Technology, Wuhan \\ 430022, China; ${ }^{2}$ National Research Council, Winnipeg, Manitoba, Canada R3B 1Y6; ${ }^{3}$ Department of Physiology, Faculty of Medicine, \\ University of Manitoba, Winnipeg, Manitoba, Canada R3E 3P5; ${ }^{4}$ Department of Cardiovascular Surgery, the First Affiliated Hospital, \\ Harbin Medical University, Harbin 150001, China; ${ }^{5}$ Cardiac Science Program, Institute of Cardiovascular Science, St Boniface General \\ Hospital, Winnipeg, Manitoba, Canada R2H 2 A6
}

\begin{abstract}
Aim: To investigate the relationship between the collateral circulation and contrast-enhanced MR signal change for myocardial infarction (MI) in pigs.

Methods: Pigs underwent permanent ligation of two diagonal branches of the left anterior descending artery. First-pass perfusion (FPP) MRI (for detecting myocardial perfusion abnormalities) and delayed enhancement (DE) MRI (for estimating myocardial infarction) using Gd-DTPA were performed at $2 \mathrm{~h}, 7 \mathrm{~d}$ and 4 weeks after the coronary occlusion. Myocardial blood flow (MBF) was evaluated using nonradioactive red-colored microspheres. Histological examination was performed to characterize the infarcts.

Results: Acute MI performed at $2 \mathrm{~h}$ afterwards was characterized by hypoenhancement in both FPP- and DE-MRI, with small and almost unchanged FPP-signal intensity (SI) and DE-SI due to negligible MBF. Subacute MI detected $7 \mathrm{~d}$ afterwards showed small but significantly increaseing FPP-SI, and was visible as a sluggish hyperenhancement in DE-MRI with considerably higher DE-SI compared to the normal myocardium; the MBF approached the half-normal value. Chronic MI detected at 4 weeks afterwards showed increasing FPP-SI comparable to the normal myocardium, and a rapid hyperenhancement in DE-MRI with even higher DE-SI; the MBF was close to the normal value. The MBF was correlated with FPP-SI $(r=+0.94, P<0.01)$ and with the peak DE-SI $(r=+0.92, P<0.01)$ at the three MI stages. Remodeled vessels were observed at intra-infarction and peri-infarction zones during the subacute and chronic periods. Conclusion: Progressive collateral recovery determines the characteristic profiles of contrast-enhanced MRI in acute, subacute and chronic myocardial infarction in pigs. The FPP- and DE-MRI signal profiles not only depend on the loss of tissue viability and enlarged interstitial space, but also on establishing a collateral circulation.
\end{abstract}

Keywords: myocardial infarction; myocardial reperfusion; contrast media; magnetic resonance imaging; gadolinium DTPA; coronary circulation; pigs; time factors

Acta Pharmacologica Sinica (2015) 36: 463-472; doi: 10.1038/aps.2014.158; published online 23 Mar 2015

\begin{abstract}
Introduction
Severe interruption of the blood supply leads to cardio myocyte necrosis that is characterized by disruption of the myocyte cell membrane. The loss of the myocyte membrane integrity allows the extracellular contrast agent to penetrate into the intracellular compartment, generating an enlarged partition coefficient of gadolinium-diethylenetriaminepentaacetic acid (Gd-DTPA) ${ }^{[1,2]}$. Consequently, the acutely infarcted myocardium appears as a hyperintense, bright
\end{abstract}

\footnotetext{
* To whom correspondence should be addressed.

E-mail jianwang1030@126.com

Received 2014-08-05 Accepted 2014-10-30
}

region on delayed gadolinium-enhanced MR imaging. With the progression of infarction remodeling, the necrotic myocardium eventually evolves into collagenous scar tissue. The pathological features of the chronic scar infarction consist of discrete collagen meshwork and loss of cellularity, leading to an enlarged interstitial space between collagen fibers. The enlarged interstitial compartment is accessible to extracellular contrast agents; therefore, chronic myocardial infarction is also associated with an increased local distribution of contrast agents ${ }^{[3-5]}$. Accordingly, chronic scar infarction is observed as hyperenhancement with delayed enhancement $\mathrm{MRI}^{[6,7]}$.

Contrast material-enhanced MR imaging depends not only on the cell membrane integrity and loss of cellularity 
but also on the patency of the vasculature that supports the infarcted myocardium as well as on the regional myocardial blood flow. An acute reperfused myocardial infarction can be identified as a central black hypoenhanced core surrounded by a bright hyperenhanced rim on delayed enhancement $\mathrm{MRI}^{[8,9]}$. An altered MR contrast enhancement pattern in the acute reperfused myocardial infarction is primarily due to the regional difference in the microvascular blood flow between the core and $\operatorname{rim}^{[8,9]}$. The hypoenhanced core on MRI is characterized by the profoundly reduced myocardial blood flow and reflects the widespread microvascular obstruction from erythrocyte stasis and neutrophil accumulation during the reperfusion period ${ }^{[10]}$. The hyperenhanced rim on MRI is mostly attributed to the restoration of reperfused blood flow, which allows contrast agents to enter into the intracellular space of dead cardiomyocytes ${ }^{[10]}$. Moreover, the regional myocardial blood flow has a critical impact on the wash-in and wash-out patterns of contrast agents. Kim et al demonstrated that the prolonged wash-out kinetics of the contrast agent is the main mechanism for the delayed hyperenhancement in acute reperfused infarction ${ }^{[11]}$.

Myocardial ischemia and infarction stimulate the development of a coronary collateral circulation, leading to a progressive increase in the myocardial blood flow that supplies the infarcted myocardium. The jeopardized myocardium in acute, subacute, and chronic infarctions does not differ significantly in terms of the loss of tissue viability and enlarged interstitial space; nonetheless, these are widely different in the histological structure and collateral circulation $^{[12,13]}$. We hypothesized that the difference in the collateral circulation among the three phases of myocardial infarctions could be responsible for the alteration in the cardiac MRI profiles during the first-pass and steady state of the contrast agent. The present study was designed to determine the potential relationship between the regional collateral blood flow and contrast-induced MR signal changes for three different stages of myocardial infarctions.

\section{Materials and methods}

This study was carried out in strict accordance with the recommendations in the Guide for the Care and Use of Laboratory Animals of National Research Council of Canada and Huazhong University of Science and Technology. The protocol was approved by the Committee on the Ethics of Animal Experiments of National Research Council of Canada and Huazhong University of Science and Technology.

\section{A pig model of myocardial infarction}

The pig heart is similar to the human heart in its size, anatomy, metabolism, and collateral circulation ${ }^{[14,15]}$. As a result, domestic pigs were chosen as the animal model for this study.

After the induction of anesthesia, eighteen domestic pigs, weighing 20-25 kg, were intubated and ventilated with gas anesthesia consisting of $1 \%-2 \%$ isoflurane in a mixture of oxygen and nitrous oxide. Using a sterile technique, a lateral thoracotomy was performed at the fourth left intercostal space to expose the heart. The pericardium was opened. The first and second diagonal branches of the left anterior descending coronary artery were permanently ligated at their origins. Complete occlusion of the vessels was confirmed by visualization of the regional myocardial cyanosis. The animals in the acute $(n=6)$, sub-acute $(n=6)$ and chronic $(n=6)$ infarction groups were imaged in an MR scanner at approximately $2 \mathrm{~h}, 7 \mathrm{~d}$, and 4 weeks after the coronary ligation procedure, respectively.

\section{Magnetic resonance imaging}

MR imaging was performed in a 3.0T MR imaging unit (Magnetom Vision; Siemens Medical System, Erlangen, Germany) equipped with a five-element cardiac phased-array coil. Stable anesthesia was maintained during the entire course of the experiment; the blood pressure and pulse rate were also monitored. Electrocardiographic signals were used to trigger MRI data acquisitions over multiple heart beats. Because all pigs underwent mechanical ventilation during the examination, a breath-hold state could easily be achieved by stopping mechanical ventilation during image acquisition.

First-pass perfusion MR imaging was used to detect myocardial perfusion abnormalities. A dose of $0.2 \mathrm{mmol}$ per kilogram of body weight of Gd-DTPA (Magnevist; Bayer Schering Pharma AG, Berlin, Germany) was administered intravenously at a rate of $3 \mathrm{~mL} / \mathrm{s}$ with a power injector (Spectris Solaris; Medrad, Warrendale, PA, USA). Immediately after injection, one image per R-R interval was acquired for the next 60 heartbeats at the apical, midventricular and basal sections along the short axis of the heart using a multislice saturationrecovery TurboFLASH sequence. The imaging parameters were as follows: repetition time, $2.4 \mathrm{~ms}$; echo time, $2.17 \mathrm{~ms}$; temporal resolution, $150.97 \mathrm{~ms}$; slice thickness, $5 \mathrm{~mm}$; field of view, $360 \times 270 \mathrm{~mm}^{2}$; and acquisition matrix, 128×96.

Delayed enhancement MR imaging was used to estimate myocardial infarction. At $8 \mathrm{~min}$ after injection, delayed enhancement MR imaging was performed in three short-axis left ventricular sections at the apical, midventricular and basal levels by an inversion-recovery prepared $\mathrm{T}_{1}$-weighted gradient echo sequence. MR images were obtained at 4-min intervals for a total of $36 \mathrm{~min}$. The imaging parameters were as follows: flip angle, 20 ; echo time, $1.56 \mathrm{~ms}$; repetition time, $564 \mathrm{~ms}$; slice thickness, $5 \mathrm{~mm}$; field of view, $225 \times 270 \mathrm{~mm}^{2}$; and acquisition matrix, $192 \times 256$. The inversion time varied from 200 to $300 \mathrm{~ms}$ according to the myocardium nulling. A recent study emphasized the importance of adjusting the inversion time after the administration of contrast agents, because the concentration of gadolinium decreased continuously after injection ${ }^{[16,17]}$.

\section{Measurement of the myocardial blood flow}

At end of the MRI sessions, approximately $5 \times 10^{6}-8 \times 10^{6}$ nonradioactive red-colored microspheres $(15 \pm 1.9 \mu \mathrm{m}$ diameters, suspended in $2 \mathrm{~mL}$ of saline solution) were injected into the left atrium. Arterial reference samples were simultaneously withdrawn from the ascending aorta 
at a constant rate of $5 \mathrm{~mL} / \mathrm{min}$ for $3 \mathrm{~min}$, starting $1 \mathrm{~min}$ before injection of the microspheres. The pig hearts were then removed from the animals under full-depth anesthesia. The hearts were sectioned along the cardiac short axis. Myocardial samples were collected from the left ventricular wall in a pie-shape. The blood samples collected from the aorta and myocardial samples were digested. The numbers of microspheres in the samples were counted using a spectrophotometer at a wavelength of $536 \mathrm{~nm}$. Blood flow was then calculated based on the microsphere count in the tissue and blood samples as well as the speed of blood collection.

\section{Histological examination}

Post-mortem tissue samples from the infarct and peri-infarct regions were examined by histopathology. The samples were fixed in $10 \%$ formalin, embedded in paraffin, and sectioned with $8-\mu \mathrm{m}$ thickness. Sections were stained with hematoxylin and eosin. Masson's trichrome stain was used to define the scarred myocardium.

\section{MR image analysis}

MRI data analysis was performed using freely available software (Segment, Version 1.8R0438, http:/ / segment.heiberg. se) with consensus between two experienced observers who were blinded to all experimental data. The images were analyzed at the end of the study to avoid issues, such as learning bias. The area of myocardial infarction was defined as the myocardium with signal intensity lower or higher than 2 standard deviations above the mean signal intensity of the remote normal myocardium ${ }^{[18]}$. A region of interest (ROI) was placed in the hypoenhanced or hyperenhanced areas, if present; left ventricular cavity and remote areas opposite of the infarct territory. The contrast ratio was calculated as the signal intensity in the infarction area divided by that in the remote area before contrast injection. The changes in the signal intensity of the delayed enhancement were plotted against time. The crosssectional areas of the infarcted myocardium were measured at the papillary muscle level in the short-axis views of delayed enhancement MRI.

The locations of these regions (the hypoenhanced region, hyperenhanced region, and remote normal myocardium and left ventricular cavity) were then transferred to the first-pass perfusion images. Care was taken to define the regions of interest several pixels from epicardial and endocardial surfaces to avoid partial volume effects. Myocardial first-pass time intensity curves were then generated within these regions for the passage of the contrast agent. The time intensity curves were normalized by expression of all signal intensity of the first-pass enhancement as a percentage of the fully relaxed intensity.

\section{Statistical analysis}

All numerical results were presented as the mean \pm standard deviation. Statistical analysis was performed using Statistica (Statsoft Inc, Tulsa, OK). Differences in the signal intensity between the normal and infarcted myocardium at specific time points were analyzed by the Bonferroni $t$ test. The changes over time in the signal intensity obtained from the infarcted myocardium were compared with the patterns obtained from the normal myocardium by repeated measures analysis of variance (ANOVA). The differences in the myocardial blood flow and infarct size were determined with a paired $t$ test. Moreover, the correlation between the myocardial blood flow and contrast-enhanced signal intensity or infarct size was determined by linear regression analysis. A $P$-value $<0.05$ indicates statistical significance.

\section{Results}

\section{Acute myocardial infarction}

Two hours after permanent coronary occlusion, a bolus injection of Gd-DTPA resulted in a substantial signal increase in both the left ventricular blood and normal myocardium (upper panel, Figure 1). Nonetheless, the signal intensity of the acutely infarcted myocardium did not change significantly during the first pass of the contrast agent (from $0.17 \% \pm 0.91 \%$ to $2.2 \% \pm 0.9 \%$ of the pre-contrast baseline level) (upper panel, Figure 1). Therefore, first-pass perfusion MRI identified
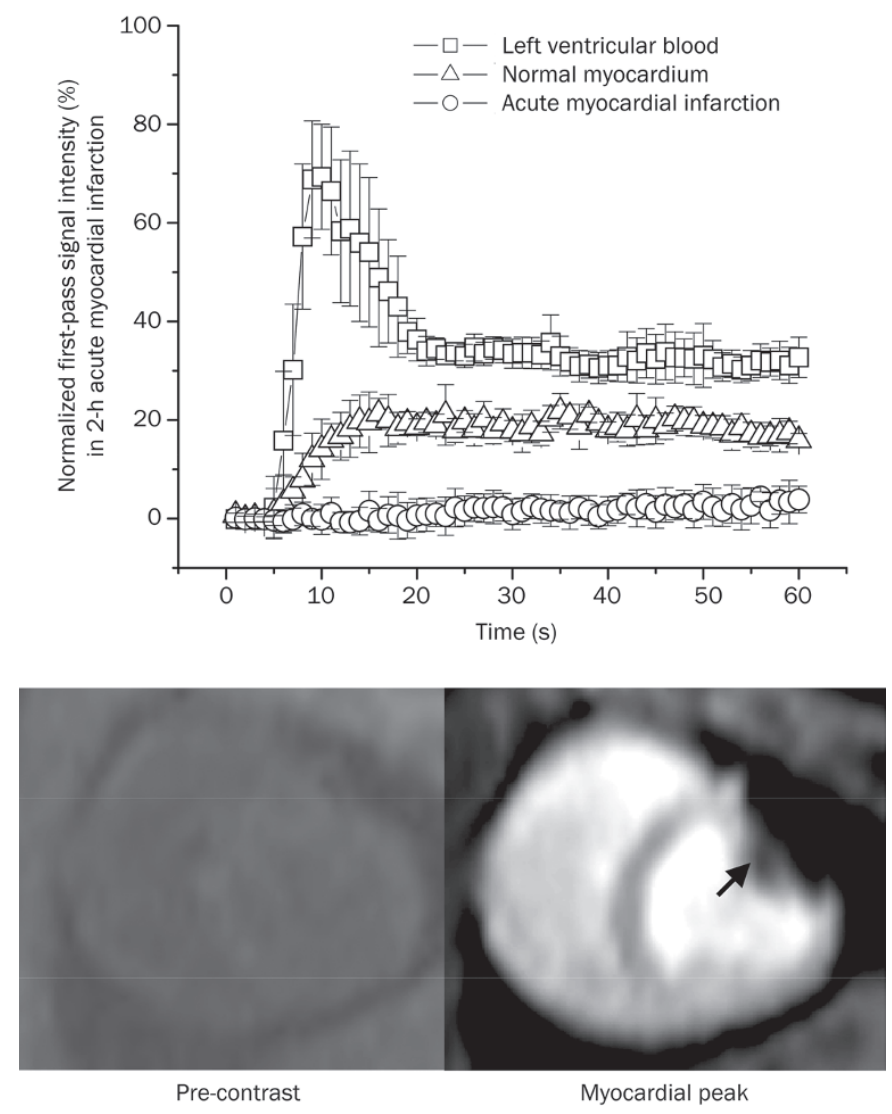

Figure 1. Time intensity curves and representative images of firstpass perfusion MRI in acute myocardial infarction. The first-pass signal intensity in acutely infarcted myocardium did not change significantly, whereas the remote myocardium had a substantial signal elevation during the first pass of the contrast agent (upper panel). Therefore, first-pass perfusion MRI identified the acute myocardial infarction as a region of perfusion defect with hypoenhancement (arrowheads, lower panel). 
acute myocardial infarction as a perfusion defect area with hypoenhancement (arrowheads, lower panel, Figure 1).

Likewise, the acutely infarcted myocardium did not have significantly increased signal intensity during the period of delayed enhancement. The delayed enhancement signal intensities in the acutely infarcted myocardium varied only from $35.9 \% \pm 27.0 \%$ to $38.1 \% \pm 23.2 \%$ of the initial baseline value for different post-contrast time points and were significantly lower than those observed in the normal myocardium, which varied from $473.5 \% \pm 36.6 \%$ to $156.1 \% \pm 29.4 \%$ of the initial baseline value (upper panel, Figure 2). Accordingly, the acutely infarcted myocardium was identified as a region of hypoenhancement relative to the normal myocardium on delayed enhancement MRI (lower panel, Figure 2). As expected, no meaningful blood flow was found in the acutely infarcted myocardium $\left(0.06 \pm 0.01 \mathrm{~mL} \cdot \mathrm{min}^{-1} \cdot \mathrm{g}^{-1}\right)$ (Figure $3 \mathrm{~A}$ ).
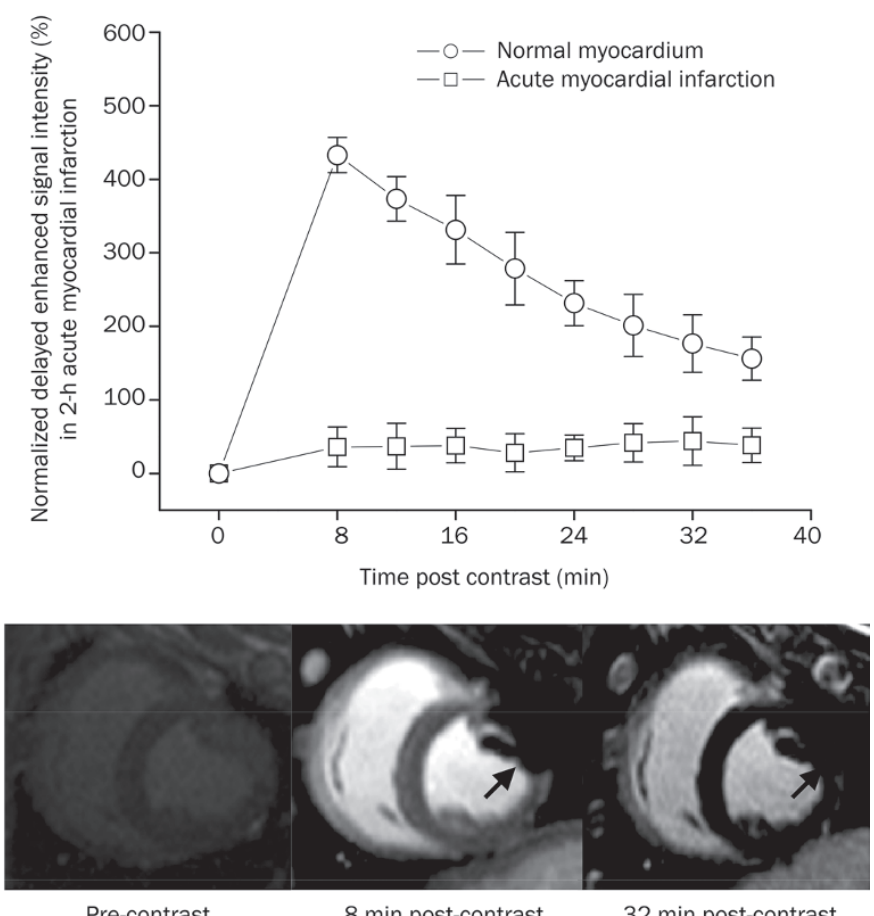

Figure 2. Time intensity curves and representative images of delayed enhancement MRI in acute myocardial infarction. The delayed enhancement signal intensity in acutely infarcted myocardium remained almost unchanged (upper panel). Therefore, the acute myocardial infarction appeared hypoenhanced relative to the normal myocardium on delayed enhancement MRI (arrowheads, lower panel).

\section{Subacute myocardial infarction}

Seven days after permanent coronary occlusion, the subacutely infarcted myocardium showed a relatively small but significant increase in signal intensity during the first pass of the contrast agent (upper panel, Figure 4). The peak firstpass signal intensity in subacutely infarcted myocardium reached $11.9 \% \pm 2.4 \%$ of the pre-contrast baseline value and was significantly higher than that observed in the acutely infarcted myocardium $(2.2 \% \pm 0.9 \%)$; nonetheless, it was still significantly lower than that in the normal myocardium $(24.8 \% \pm 3.6 \%)$ (upper panel, Figure 4).

The delayed enhancement signal intensity in the subacutely infarcted myocardium varied from $1048.3 \% \pm 84.5 \%$ to $701.4 \% \pm 79.9 \%$ of the initial baseline value for different postcontrast time points and was considerably higher than that in the normal myocardium, which varied from $459.2 \% \pm 68.6 \%$ to $192.4 \% \pm 61.8 \%$ of the initial baseline value (upper panel, Figure 5). Therefore, the subacutely infarcted myocardium was observed as a hyperenhanced zone on delayed enhancement MRI (arrowheads, lower panel, Figure 5). Moreover, the infarcted epicardium had an earlier hyperenhancement than the infarcted endocardium. The infarcted epicardium acquired the hyperenhancement at 16 min post-contrast; while transmural delayed hyperenhancement was ultimately observed at 32 min post-contrast. The subacutely infarcted myocardium also showed a certain degree of regional myocardial blood flow $\left(0.46 \pm 0.15 \mathrm{~mL} \cdot \mathrm{min}^{-1} \cdot \mathrm{g}^{-1}\right)$, which was considerably higher than that in the acutely infarcted myocardium $(0.06 \pm 0.01$ $\mathrm{mL} \cdot \mathrm{min}^{-1} \cdot \mathrm{g}^{-1}$ ) (Figure 3A).

\section{Chronic myocardial infarction}

Four weeks after permanent coronary occlusion, a bolus injection of Gd-DTPA resulted in a rapid and uniform signal increase in both the chronic infarction and normal myocardium during the first pass of the contrast agent (upper panel, Figure 6). The first-pass signal intensity in the chronic infarction and normal myocardium increased from $5.6 \% \pm 1.3 \%$ to $25.2 \% \pm 3.4 \%$ and from $6.2 \% \pm 1.5 \%$ to $28.3 \% \pm 3.8 \%$ relative to the baseline value, respectively (upper panel, Figure 6). Therefore, the first-pass perfusion MRI may be unable to distinguish between the chronic infarction and normal myocardium (lower panel, Figure 6).

The delayed enhancement signal intensity in the chronically infarcted myocardium varied from $1455.8 \% \pm 83.5 \%$ to $997.2 \% \pm 83.3 \%$ of the initial baseline value for different postcontrast time points and was considerably higher than that in the normal myocardium, which varied from $447.9 \% \pm 51.5 \%$ to $199.1 \% \pm 65.5 \%$ of the initial baseline value (upper panel, Figure 7). Thus, the chronically infarcted myocardium was identified as the bright region of hyperenhancement on delayed enhancement MRI (arrowheads, lower panel, Figure 7). Transmural delayed hyperenhancement was rapidly achieved at 16 min post-contrast. The regional myocardial blood flow in the chronically infarcted myocardium $\left(0.91 \pm 0.10 \mathrm{~mL} \cdot \mathrm{min}^{-1} \cdot \mathrm{g}^{-1}\right)$ was markedly higher than that in the subacutely infarcted myocardium $\left(0.46 \pm 0.15 \mathrm{~mL} \cdot \mathrm{min}^{-1} \cdot \mathrm{g}^{-1}\right)$ and not significantly different from that in the normal myocardium $(1.05 \pm 0.11$ $\mathrm{mL} \cdot \mathrm{min}^{-1} \cdot \mathrm{g}^{-1}$ ) (upper and left panel, Figure 3).

\section{Correlation between the myocardial blood flows and contrast-} induced MR signal changes

The regional myocardial blood flows were $0.06 \pm 0.01,0.46 \pm 0.15$, and $0.91 \pm 0.10 \mathrm{~mL} \cdot \mathrm{min}^{-1} \cdot \mathrm{g}^{-1}$ in the acutely, subacutely and chronically infarcted myocardium, respectively (upper and 

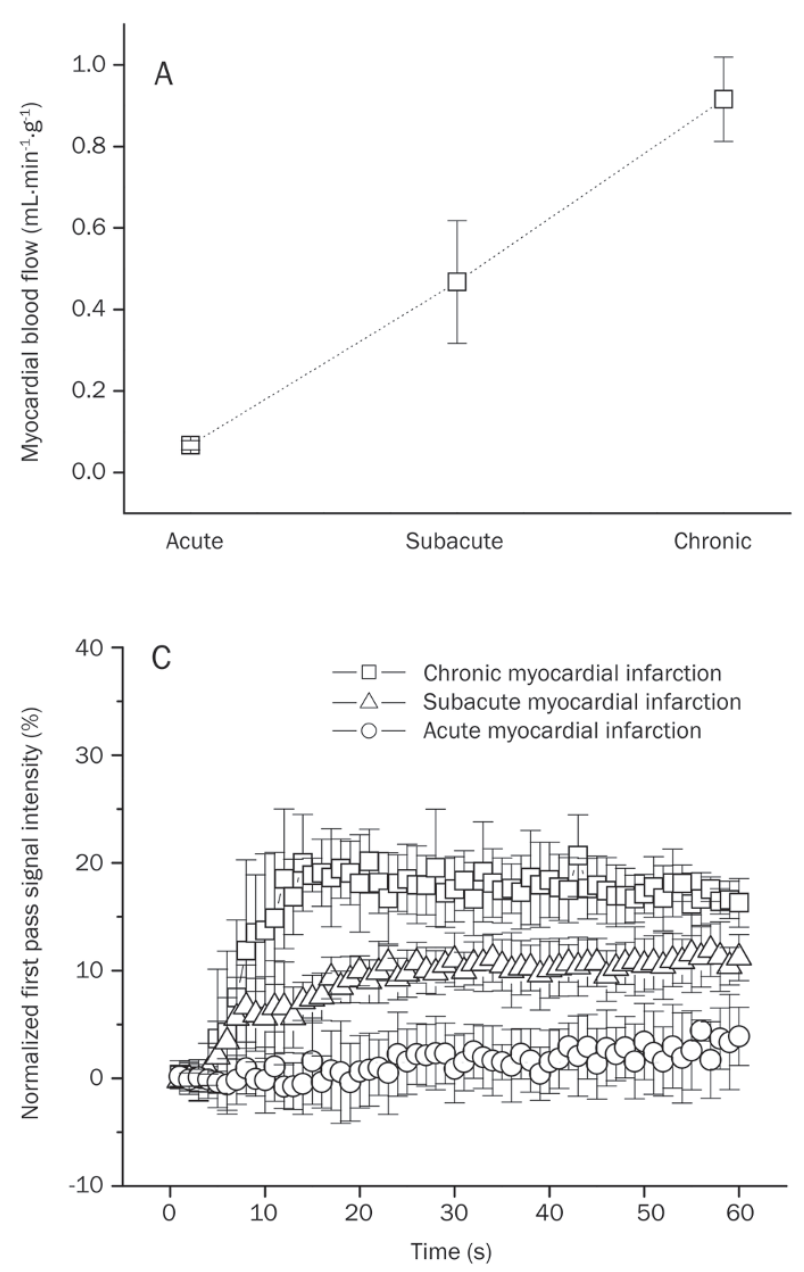
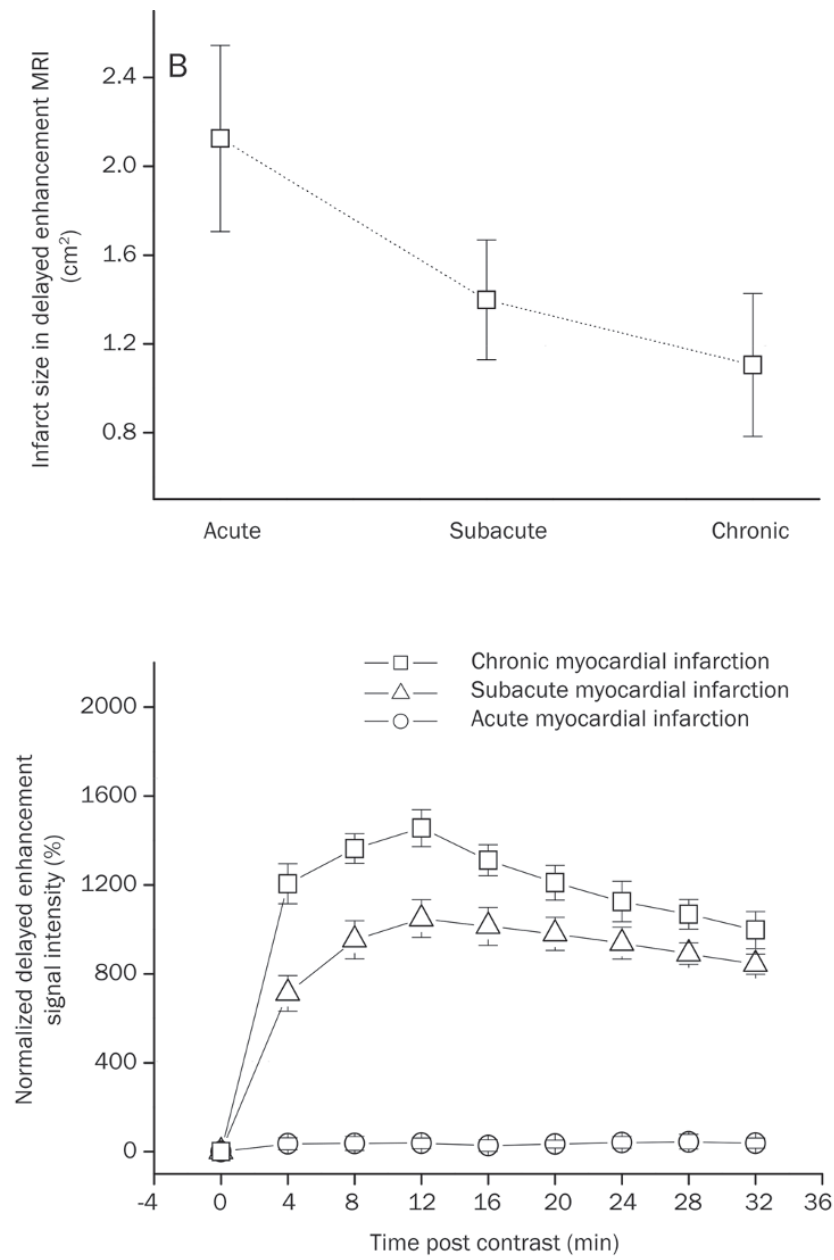

Figure 3. The myocardial blood flow, infarct size, first-pass perfusion and delayed enhancement signal intensity in acute, subacute, and chronic infarctions. The regional myocardial blood flows in the infarcted areas were progressively recovered with the evolution of the myocardial infarction (A). The infarct size tended to decrease with the development of post-infarction remodeling (B). Interestingly, there were gradual increases in the peak signal intensities for both first-pass perfusion and delayed enhancement (C).

left panel, Figure 3). The cross-sectional areas of the infarcted myocardium were $2.12 \pm 0.42,1.39 \pm 0.27$, and $1.11 \pm 0.32 \mathrm{~cm}^{2}$ in acute, subacute and chronic infarction, respectively (upper and right panel, Figure 3), and the area covered by the infarcted myocardium showed a trend of decreasing with the progressive collateral circulation recovery during postinfarction remodeling. Interestingly, the peak first-pass signal intensities in the infarcted regions also increased gradually from $2.2 \% \pm 0.9 \%$ of the pre-contrast baseline value for acute infarction, to $11.1 \% \pm 2.4 \%$ for sub-acute infarction, and ultimately to $20.1 \% \pm 2.9 \%$ for chronic infarction (lower and left panel, Figure 3). Moreover, the peak signal intensities of delayed enhancement in the infarcted regions were significantly and progressively elevated from $38.1 \% \pm 23.2 \%$ of the initial baseline value for acute infarction, to $1048.3 \% \pm 84.4 \%$ for subacute infarction, and eventually to $1455.8 \% \pm 83.5 \%$ for chronic infarction (lower and right panel, Figure 3).

More importantly, the myocardial blood flows in the infarcted myocardium were positively correlated with the peak signal intensities during the first-pass $(r=0.94, P<0.01)$ (top panel, Figure 8) and delayed enhancement $(r=0.92$, $P<0.01$ ) (middle panel, Figure 8). The results of this study also showed a strong negative correlation between the regional myocardial blood flows and cross-sectional areas of the infarcted myocardium $(r=-0.82, P<0.01)$ (bottom panel, Figure 8).

\section{Histological characteristics of the infarcted myocardium}

Inflammatory cell infiltration and intramyocardial hemorrhage, suggesting microvascular damage, were observed in the acute myocardial infarctions (black arrowheads, top panel, Figure 9). In the subacute myocardial infarctions, the myocytes were replaced by granulation tissue (white arrowheads) and early collagen protein content; large thick-walled blood vessels were apparent at the peri-infarction zone with small areas of interstitial fibrosis (black arrowheads, middle panel, Figure 9). The chronic myocardial infarctions contain dense collagen with numerous intact microvessels (black arrow- 

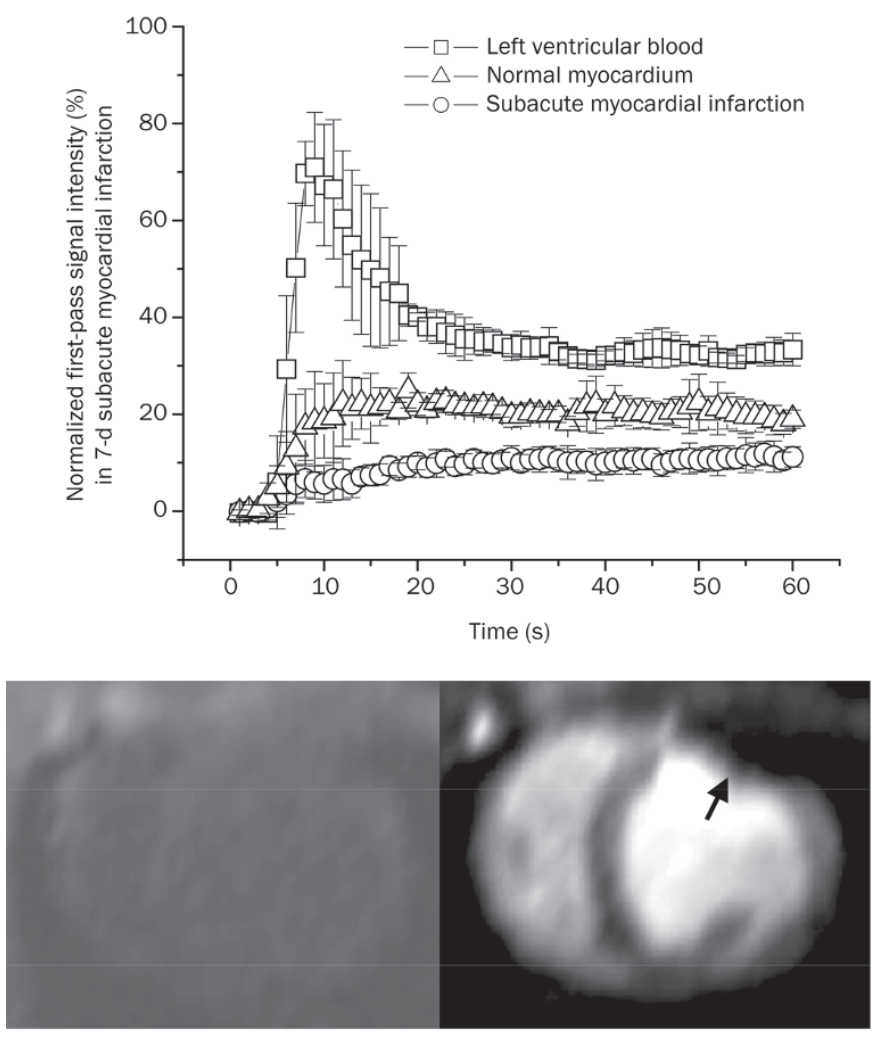

Pre-contrast

Myocardial peak

Figure 4. Time intensity curves and representative images of the firstpass perfusion MRI in subacute myocardial infarction. The first-pass signal intensity in subacute infarction had a degree of elevation that was still lower than that in the normal myocardium. Therefore, the subacute myocardial infarction was identified as a region of hypoenhancement on first-pass perfusion MRI (arrowheads, lower panel).

heads, bottom panel, Figure 9). The remodeled vessels in healing infarctions were likely responsible for the re-establishment of tissue perfusion that supports contrast transportation.

\section{Discussion}

The first-pass signal intensity could be plotted as a function of time to characterize the transmit kinetics of contrast material. The analysis of time intensity curves and first-pass images could reflect regional variations in the myocardial blood flow ${ }^{[19,20]}$. The extent of a signal increase as a function of the input function correlates well with the microsphere blood flow measurement ${ }^{[21]}$. A delayed enhancement image, on the other hand, highlights the infarcted myocardium with the increased distribution volume or coefficient of contrast agents $^{[1,2]}$. The distribution coefficient of contrast agents in the injured myocardium depends on the cell membrane integrity and the volume of interstitial space ${ }^{[3-5]}$. Pathological processes sequentially undergo extensive cardiomyocyte necrosis, granulation tissue formation and collagenous scar remodeling at three different stages of healing infarctions, which are associated with the loss of tissue viability and enlarged interstitial tissue that is suitable for the contrast accumulation. There-
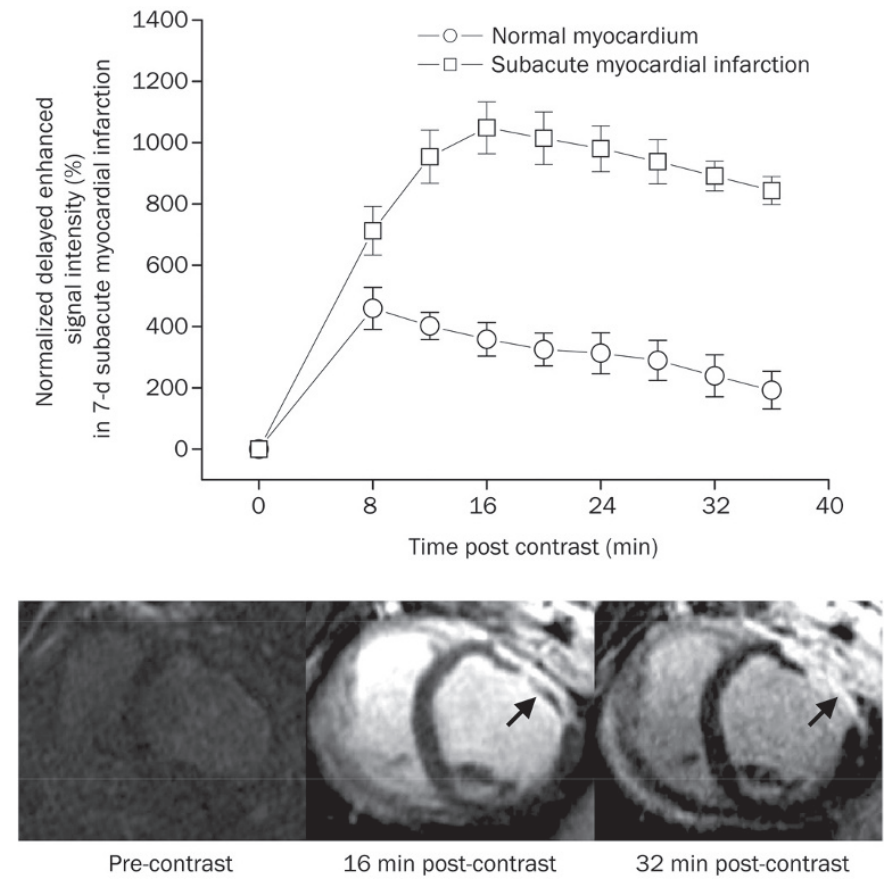

Figure 5. Time intensity curves and representative images of delayed enhancement MRI in subacute myocardial infarction. The delayed enhancement signal intensity in the subacute infarction was significantly higher than that in the normal myocardium (upper panel). The infarcted epicardium displayed an earlier hyperenhancement than the infarcted endocardium. The infarcted epicardium acquired hyperenhancement at 16 min post-contrast, while transmural hyperenhancement was ultimately observed at 32 min post-contrast (arrowheads, lower panel).

fore, collateral recovery is another critical factor affecting the contrast material-enhanced MR patterns at the three different stages of healing infarctions after permanent coronary occlusion.

Lack of collateral flow and the hypoenhancement of first-pass perfusion and delayed enhancement MRIs in acute myocardial infarction

The acutely infarcted myocardium did not show an increase in signal intensity on either first-pass perfusion or delayed enhancement MRIs in spite the presence of irreversibly injured myocardium. The hypoenhancement in the acute myocardial infarction was mainly due to the lack of meaningful myocardial blood flow for delivering contrast agents to the infarcted region. The histological findings included inflammatory cell infiltration and intramyocardial hemorrhage, which were indicative of microvascular damage. The "no reflow" phenomenon in acute occlusive myocardial infarction was also demonstrated with the colored microsphere technique. Lee et al have demonstrated that myocardial ischemia caused by the occlusion of a cat coronary artery is hypoenhanced relative to the normal myocardium ${ }^{[22]}$. Wang et al demonstrated that the nonreperfused occlusive infarct exhibits a first-pass perfusion defect without any delayed hyperenhancement at $4 \mathrm{~h}$ after coronary occlusion $^{[23]}$. Thus, the disappearance of delayed 

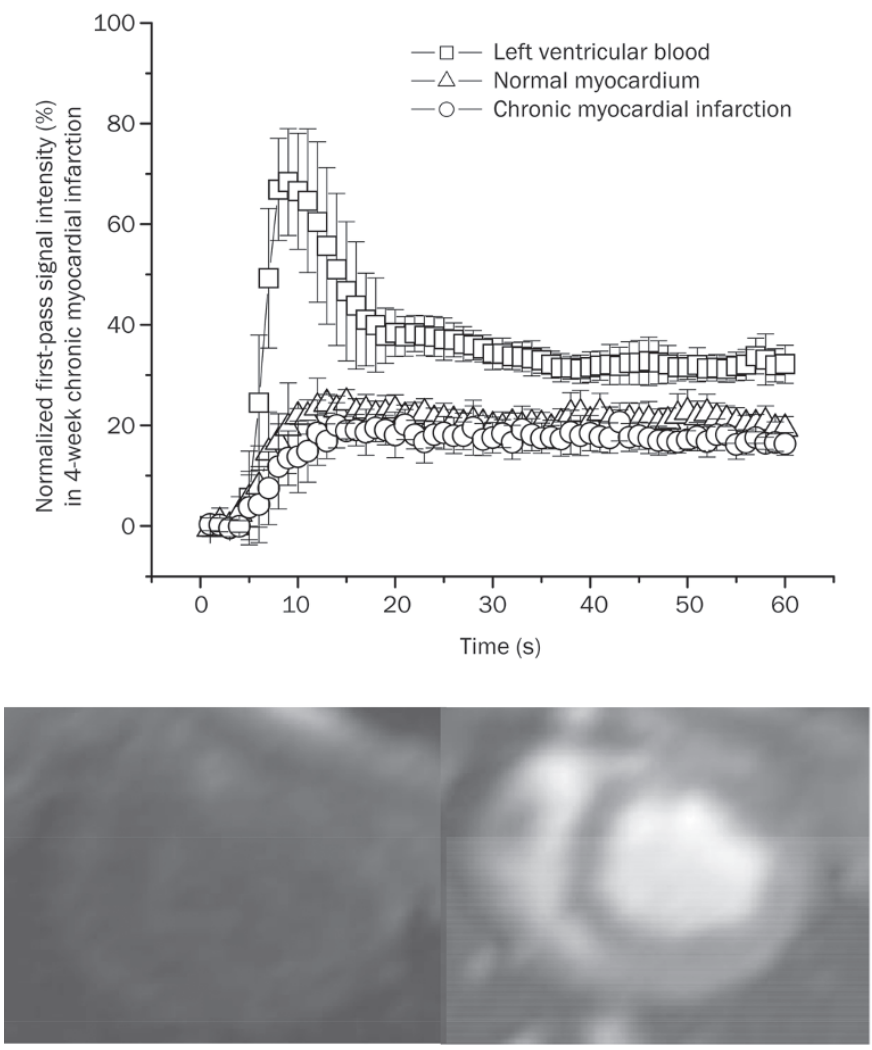

Pre-contrast

Myocardial peak

Figure 6. Time intensity curves and representative images of first-pass perfusion MRI in chronic myocardial infarction. The first-pass signal intensity in chronic infarction was not significantly different from that in normal myocardium (upper panel). Therefore, first-pass perfusion MRI could not distinguish between chronic infarction and the normal myocardium (lower panel).

hyperenhancement is not necessarily an indication of viable myocardium. The signal changes of first-pass perfusion and delayed enhancement do not depend on the loss of myocardial viability; instead, they depend on the regional myocardial blood flow in an acute occlusive myocardial infarction.

The partially restored collateral flow and sluggish hyperenhancement in subacute myocardial infarction

In 7-d subacute myocardial infarction, a bolus injection of contrast agents resulted in a relatively small but significant elevation on first-pass signal intensity in the infarcted myocardium even though the magnitude of the signal increase in the infarcted myocardium was less pronounced than in the normal myocardium. The subacute myocardial infarction had some collateral circulation $\left(0.46 \pm 0.15 \mathrm{~mL} \cdot \mathrm{min}^{-1} \cdot \mathrm{g}^{-1}\right)$, reaching approximately half of the myocardial blood flow in the normal myocardium. Large, thick-wall vessels were present at the peri-infarct zone in subacutely infarcted myocardium. Partially restored myocardial blood flow through newly developed collateral vessel has facilitated delivery of contrast agents into the subacutely infarcted myocardium, resulting in some delayed hyperenhancement. The hyperenhancement
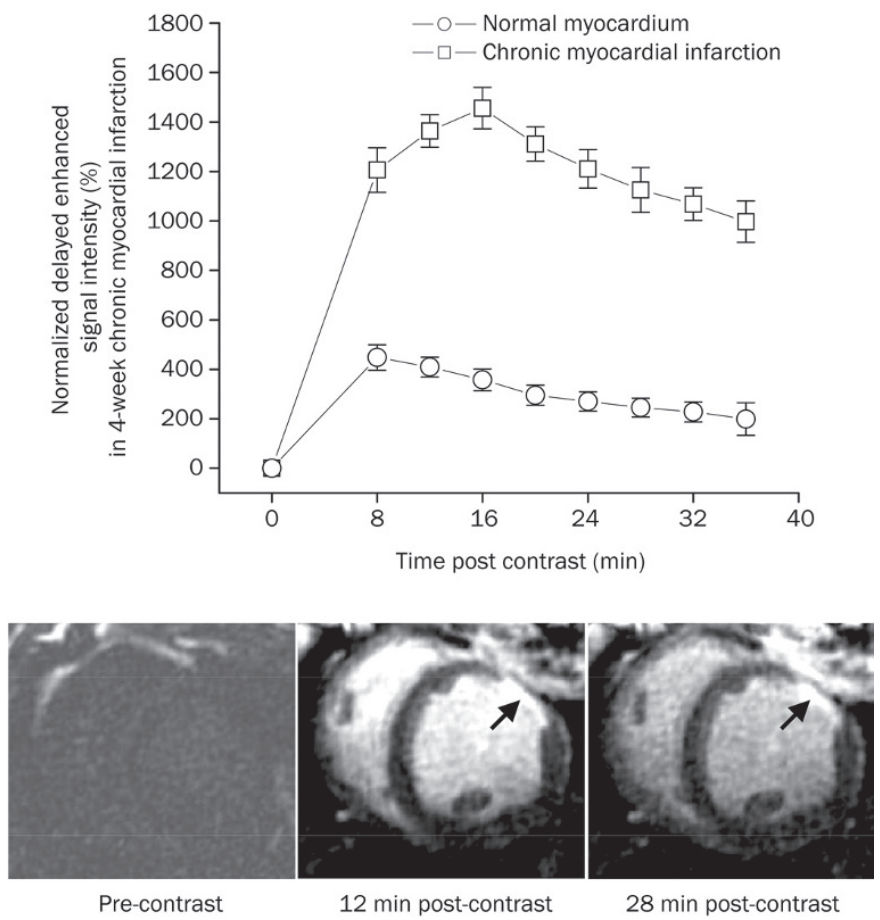

Figure 7. Time intensity curves and representative images of delayed enhancement MRI in chronic myocardial infarction. Delayed enhancement signal intensity in chronic infarction was significantly higher than that in the normal myocardium (upper panel). Therefore, the chronic infarction was rapidly hyperenhanced at $16 \mathrm{~min}$ after contrast injection (arrowheads, lower panel).

was faster in infarcted epicardium than in infarcted endocardium. The infarcted epicardium acquired the delayed hyperenhancement at 16 min post-contrast, while transmural hyperenhancement was ultimately achieved at $32 \mathrm{~min}$ post-contrast. The discrepancy in the delayed enhancement between these two infarcted regions might be attributed to the heterogeneous collateral circulation. It has been demonstrated that coronary collateral vessels within the epicardium can anastomose with both extracardiac vessels originating outside of the heart and intracardiac coronary vessels branching from the other coronary artery ${ }^{[24,25]}$. Conversely, the endocardial collateral circulation relies primarily on the intracoronary anastomosis ${ }^{[24,25]}$. Therefore, the subacute myocardial infarction is identified as a region of sluggish hyperenhancement on delayed enhancement MRI.

\section{Nearly normal collateral flow and the rapid hyperenhancement} in chronic myocardial infarction

In 4-week chronic myocardial infarction, a bolus injection of Gd-DTPA resulted in a similar signal increase in both infarcted and normal myocardium. Our previous study also demonstrated that the bolus of extracellular or intravascular contrast agents caused a rapid and dramatic decline in the first-pass $\mathrm{T}_{2}{ }^{*}$ signal intensity in both the scarred and normal myocardium ${ }^{[26]}$. These findings strongly indicate that the occurrence and development of collateral circulation results in a substantial res- 

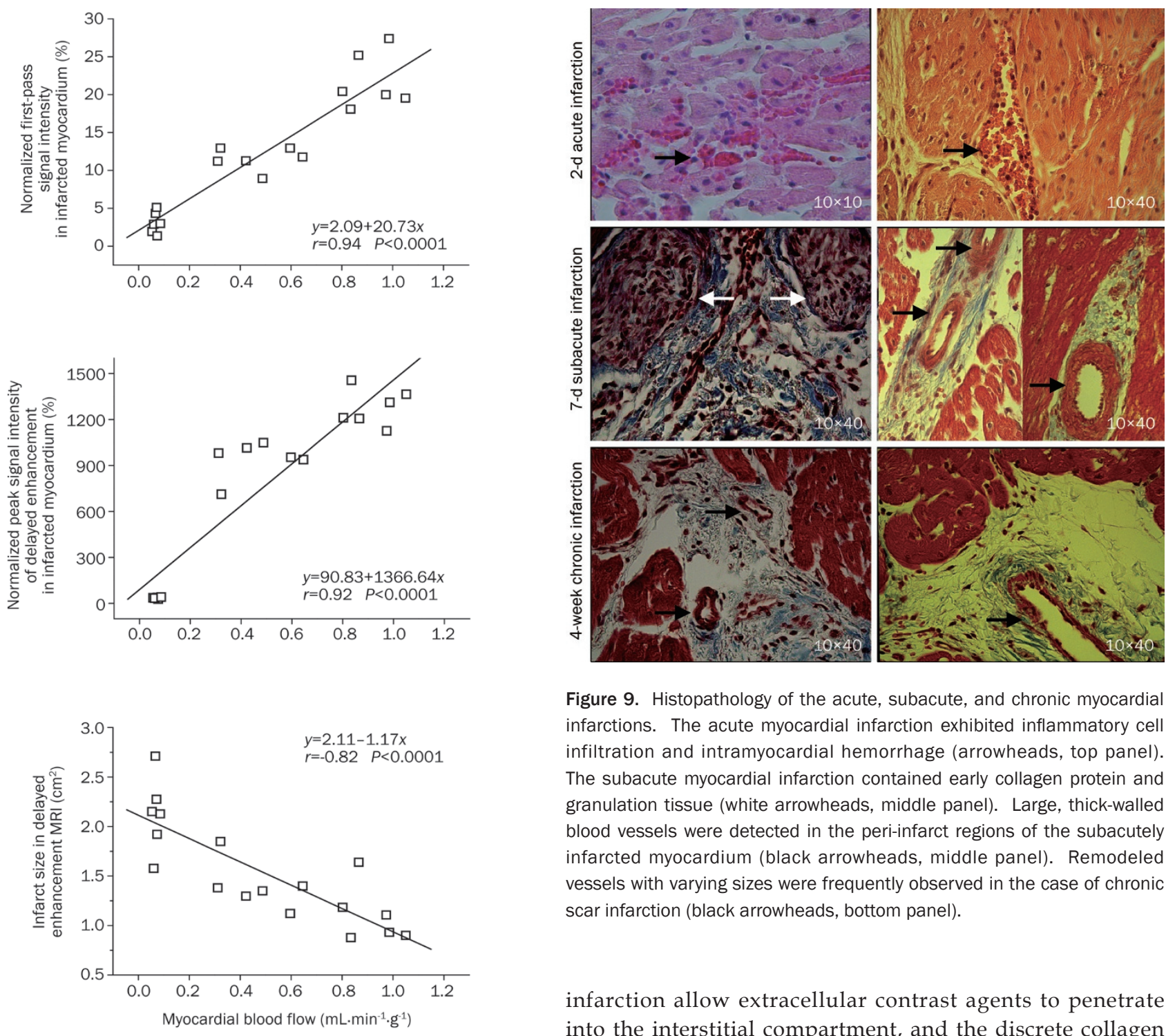

Figure 9. Histopathology of the acute, subacute, and chronic myocardial infarctions. The acute myocardial infarction exhibited inflammatory cell infiltration and intramyocardial hemorrhage (arrowheads, top panel). The subacute myocardial infarction contained early collagen protein and granulation tissue (white arrowheads, middle panel). Large, thick-walled blood vessels were detected in the peri-infarct regions of the subacutely infarcted myocardium (black arrowheads, middle panel). Remodeled vessels with varying sizes were frequently observed in the case of chronic scar infarction (black arrowheads, bottom panel).

infarction allow extracellular contrast agents to penetrate into the interstitial compartment, and the discrete collagen fiber meshwork and loss of cellularity enlarged the interstitial

Figure 8. Correlations between the myocardial blood flows and peak MR signal intensities or infarct sizes. The regional myocardial blood flows, infarct sizes and MR signal intensities were obtained in the three stages of myocardial infarctions. A strong correlation between the regional blood flow and peak signal intensity was noted in the infarcted myocardium for both first-pass perfusion MRI $(r=0.94, P<0.01)$ (top panel) and delayed enhancement MRI $(r=0.92, P<0.01)$ (middle panel). Moreover, regional myocardial blood flows were negatively correlated with the infarct areas of the pig hearts $(r=-0.82, P<0.01)$ (bottom panel).

toration of regional myocardial blood flow in chronic infarction. Masson's trichrome staining showed that the scar tissue consisted of dense collagen, massive residual capillaries, and dilated vessels. Microsphere measurements also showed that regional myocardial blood flow in chronically infarcted myocardium was comparable to that in the normal myocardium. Massive contrast agents could be rapidly delivered into the chronically infarcted myocardium through numerous, uniformly distributed vessels. Microvessels within the scar space that is accessible to extracellular contrast agents ${ }^{[27]}$. As a result, a rapid homogeneous hyperenhancement was apparent in chronic myocardial infarction on delayed enhancement MRI. Many investigators have also observed that chronic scar infarction is associated with hyperenhancement after contrast injection $^{[6,7]}$. The hyperenhancement is closely related to the elevated concentration and increased distribution volume of gadolinium contrast within a chronic scar infarction relative to the normal myocardium ${ }^{[3-5]}$. Previous studies have shown that the pig hearts develop a significant collateral network with a substantial increase in collateral flow after 3 to 4 weeks of progressive coronary artery occlusion ${ }^{[15,24]}$. An animal study that was similar to ours reported that the collateral flow supporting the infarction scar increases dramatically at 4 weeks after permanent coronary artery occlusion ${ }^{[28]}$.

\section{Conclusion}

In this study, 2-h acute, 7-d subacute, and 4-week chronic myocardial infarctions were selected as representative phases 
of infarction healing. The nonreperfused occlusive myocardial infarction sequentially underwent three distinctive stages of regional myocardial blood flow, starting from a lack of collateral flow for acute infarction, followed by partially restored collateral flow for subacute infarction and, finally, near-normal collateral flow for chronic infarction. Accordingly, delayed enhancement MRI revealed hypoenhancement, sluggish hyperenhancement and rapid hyperenhancement in acute, subacute, and chronic myocardial infarctions, respectively. We conclude that the contrast agent-enhanced MRI signal intensity depends not only on the loss of tissue viability and enlarged interstitial space, it also depends on the establishment of a collateral circulation.

\section{Acknowledgements}

We would like to acknowledge the funding support provided by the National Natural Science Foundation of China (No 81200105), the China Postdoctoral Science Foundation (No 20100470050), the Canadian Institute of Health Research (CIHR), and the National Research Council of Canada (NRC).

\section{Author contribution}

All authors participated in the design and execution of the experiments, the analysis and interpretation of the data, and the drafting and critical evaluation of the manuscript. All authors have read and approved the submitted manuscript.

\section{References}

1 Klein C, Schmal TR, Nekolla SG, Schnackenburg B, Fleck E, Nagel E. Mechanism of late gadolinium enhancement in patients with acute myocardial infarction. J Cardiovasc Magn Reson 2007; 9: 653-8.

2 Pereira RS, Prato FS, Wisenberg G, Sykes J. The determination of myocardial viability using Gd-DTPA in a canine model of acute myocardial ischemia and reperfusion. Magn Reson Med 1996; 36: 68493.

3 Pereira RS, Prato FS, Lekx KS, Sykes J, Wisenberg G. Contrastenhanced MRI for the assessment of myocardial viability after permanent coronary artery occlusion. Magn Reson Med 2000; 44: 309-16.

4 Rehwald WG, Fieno DS, Chen EL, Kim RJ, Judd RM. Myocardial magnetic resonance imaging contrast agent concentrations after reversible and irreversible ischemic injury. Circulation 2002; 105 : 224-9.

5 Klein C, Nekolla SG, Balbach T, Schnackenburg B, Nagel E, Fleck E, et al. The influence of myocardial blood flow and volume of distribution on late Gd-DTPA kinetics in ischemic heart failure. J Magn Reson Imaging 2004; 20: 588-93.

6 Kirschner R, Toth L, Varga-Szemes A, Simor T, Suranyi P, Kiss P, et al. Differentiation of acute and four-week old myocardial infarct with Gd(ABE-DTTA)-enhanced CMR. J Cardiovasc Magn Reson 2010; 12: 22.

7 Abdel-Aty H, Zagrosek A, Schulz-Menger J, Taylor AJ, Messroghli D, Kumar A, et al. Delayed enhancement and T2-weighted cardiovascular magnetic resonance imaging differentiate acute from chronic myocardial infarction. Circulation 2004; 109: 2411-6.

8 Judd RM, Lugo-Olivieri $\mathrm{CH}$, Arai M, Kondo T, Croisille P, Lima JA, et al. Physiological basis of myocardial contrast enhancement in fast magnetic resonance images of 2-day-old reperfused canine infarcts.
Circulation 1995; 92: 1902-10.

9 Rochitte CE, Lima JA, Bluemke DA, Reeder SB, McVeigh ER, Furuta T, et al. Magnitude and time course of microvascular obstruction and tissue injury after acute myocardial infarction. Circulation 1998; 98 : 1006-14.

10 Bremerich J, Wendland MF, Arheden H, Wyttenbach R, Gao DW, Huberty JP, et al. Microvascular injury in reperfused infarcted myocardium: noninvasive assessment with contrast-enhanced echoplanar magnetic resonance imaging. J Am Coll Cardiol 1998; 32: 787-93.

11 Kim RJ, Chen EL, Lima JA, Judd RM. Myocardial Gd-DTPA kinetics determine MRI contrast enhancement and reflect the extent and severity of myocardial injury after acute reperfused infarction. Circulation 1996; 94: 3318-26.

12 Yang Y, Graham JJ, Connelly K, Foltz WD, Dick AJ, Wright GA. MRI manifestations of persistent microvascular obstruction and acute left ventricular remodeling in an experimental reperfused myocardial infarction. Quant Imaging Med Surg 2012; 2: 12-20.

13 Mather AN, Fairbairn TA, Artis NJ, Greenwood JP, Plein S. Timing of cardiovascular MR imaging after acute myocardial infarction: effect on estimates of infarct characteristics and prediction of late ventricular remodeling. Radiology 2011; 261: 116-26.

14 Hearse DJ. Species variation in the coronary collateral circulation during regional myocardial ischaemia: a critical determinant of the rate of evolution and extent of myocardial infarction. Cardiovasc Res 2000; 45: 213-9.

15 Roth DM, Maruoka Y, Rogers J, White FC, Longhurst JC, Bloor CM. Development of coronary collateral circulation in left circumflex ameroid-occluded swine myocardium. Am J Physiol 1987; 253: H1279-88.

16 Simonetti OP, Kim RJ, Fieno DS, Hillenbrand HB, Wu E, Bundy JM, et al. An improved MR imaging technique for the visualization of myocardial infarction. Radiology 2001; 218: 215-23.

17 Lund GK, Stork A, Saeed M, Bansmann MP, Gerken JH, Müller V, et al. Acute myocardial infarction: evaluation with first-pass enhancement and delayed enhancement MR imaging compared with 201TI SPECT imaging. Radiology 2004; 232: 49-57.

18 Gerber BL, Garot J, Bluemke DA, Wu KC, Lima JA. Accuracy of contrast-enhanced magnetic resonance imaging in predicting improvement of regional myocardial function in patients after acute myocardial infarction. Circulation 2002; 106: 1083-9.

19 Wilke N, Simm C, Zhang J, Ellermann J, Ya X, Merkle H, et al. Contrastenhanced first pass myocardial perfusion imaging: correlation between myocardial blood flow in dogs at rest and during hyperemia. Magn Reson Med 1993; 29: 485-97.

20 Su MY, Yang KC, Wu CC, Wu YW, Yu HY, Tseng RY, et al. First-pass myocardial perfusion cardiovascular magnetic resonance at 3 Tesla. J Cardiovasc Magn Reson 2007; 9: 633-44.

21 Wilke N, Jerosch-Herold M, Wang Y, Huang Y, Christensen BV, Stillman AE, et al. Myocardial perfusion reserve: assessment with multisection, quantitative, first-pass MR imaging. Radiology 1997; 204: 373-84.

22 Lee SS, Goo HW, Park SB, Lim CH, Gong G, Seo JB, et al. MR imaging of reperfused myocardial infarction: comparison of necrosis-specific and intravascular contrast agents in a cat model. Radiology 2003; 226: 739-47

23 Wang Y, Sun W, Cao G, Meng L, Song L, Du X. Delayed hyperenhancement patterns in occlusive and reperfused myocardial infarcts during different healing stages. J Magn Reson Imaging 2006; 24: 851-7.

24 Görge G, Schmidt T, Ito BR, Pantely GA, Schaper W. Microvascular and collateral adaptation in swine hearts following progressive coronary artery stenosis. Basic Res Cardiol 1989; 84: 524-35. 
25 Bloor CM, Liebow AA. Coronary collateral circulation. Am J Cardiol 1965; 16: 238-52.

26 Wang J, Liu HY, Lv H, Xiang B, Gruwel M, Tomanek B, et al. Identification of chronic myocardial infarction with extracellular or intravascular contrast agents in magnetic resonance imaging. Acta Pharmacol Sin 2008; 29: 65-73.

27 Wang J, Xiang B, Lin HY, Liu H, Freed D, Arora RC, et al. Pathological mechanism for delayed hyperenhancement of chronic scarred myocardium in contrast agent enhanced magnetic resonance imaging. PLoS One 2014; 9: e96463.

28 White FC, Carroll SM, Magnet A, Bloor CM. Coronary collateral development in swine after coronary artery occlusion. Circ Res 1992; 71: 1490-500. 